\title{
DETERMINATION OF ELEMENT ABUNDANCES IN PLANETARY NEBULAE FROM RECOMBINATION LINE SPECTRA
}

\author{
A.A. NIKITIN and A.F. KHOLTYGIN \\ S.-Petersburg Univ. Astron. Obs., 198904, Petergof, Russia \\ and \\ A.A. SAPAR and T.KH. FEKLISTOVA \\ Tartu Astrophysical Observatory, EE2444, Tõravere, Estonia
}

\begin{abstract}
Carbon, nitrogen and oxygen abundances have been found from the recombination lines for 63 planetary nebulae.

Intensities of the recombination lines give important information about ion abundances in planetary nebulae. The results of our calculations of the effective recombination coefficients and the relative line intensities for ions CII, CIII, CIV, NIII, NIV, OIV and OV [1] are presented. The abundance ratio of the ions $\mathrm{A}^{+}$and $\mathrm{H}^{+}$is defined by [1]:
\end{abstract}

$$
\frac{N\left(A^{+}\right)}{N\left(H^{+}\right)} \equiv\left\{A^{+} / H^{+}\right\}=\frac{\lambda_{k i}}{\lambda\left(H_{\beta}\right)} \frac{\alpha^{e f f}\left(H_{\beta}\right)}{\alpha_{k i}^{e f f}}=X\left(T_{e}\right) \frac{I_{k i}}{I\left(H_{\beta}\right)},
$$

where $I_{k i}$ is the observed line intensity for transition $k \rightarrow i$ corrected for interstellar extinction and $I\left(H_{\beta}\right)$ is the same for $H_{\beta}$, the quantities $\alpha_{k i}^{e f f}$ and $\alpha^{e f f}\left(H_{\beta}\right)$ are the corresponding effective recombination coefficients. Both the radiation and dielectronic recombination contribution have been taken into account in $X\left(T_{e}\right)$. The values of $X\left(T_{\mathrm{e}}\right)$ for ions CII, CIII, CIV, NII, NIII, NIV, OII, OIII, OIV and OV are given in table. Using the ionization correction factors given in [1] the total ion abundances for $\mathrm{C}, \mathrm{N}$ and $\mathrm{O}$ have been obtained. We have determined the abundances of $\mathrm{CNO}$ elements in 63 planetary nebulae. The abundances found from the recombination line intensities are often much higher than those from collisionally excited lines.

\section{References}

Golovatyj V.V., Sapar A.A, Feklistova T.Kh., Kholtygin A.F. (1991), Atomic data for the spectroscopy of rarefied astrophysical plasma, Tartu Astr. Obs. Teated No 109, Tallinn 